\title{
REVIEWS
}

\section{A New Framework of Polymer Solution Science. The Helical Wormlike Chain}

\author{
Hiromi YamaKawA ${ }^{\dagger}$ \\ Department of Polymer Chemistry, Kyoto University, \\ Kyoto 606-8501, Japan
}

(Received October 29, 1998)

\begin{abstract}
The object of this review article is to introduce and digest Yamakawa's new book, "Helical Wormlike Chains in Polymer Solutions." A brief description is given of a new model for polymer chains, called the helical wormlike chain, and its applications to dilute solution behavior of polymers, that is, equilibrium, steady-state transport, and dynamical properties. The description follows most of the chapters of the book. It is shown that all theoretical and experimental investigations on the basis of this model provide a new framework of polymer solution science, which takes the place of the Flory-Kirkwood framework.
\end{abstract}

KEY WORDS Helical Wormlike Chain / Mean-Square Radius of Gyration / Scattering Function / Transport Coefficients / Expansion Factors / Second Virial Coefficient / Dynamic Structure Factor /

The object of this review article is to introduce and digest Yamakawa's new book ${ }^{1}$ entitled " Helical Wormlike Chains in Polymer Solutions." As stated in its Preface, it is intended to give a comprehensive and systematic description of the statistical-mechanical, transport, and dynamic theories of dilute solution properties of both flexible and semiflexible polymers, including oligomers, developed on the basis of the "helical wormlike (HW) chain" model, along with an analysis of extensive experimental data. Much of the material in the book arises from his research reported since the year of publication of his earlier (1971) book. ${ }^{2}$ The results of this research were already very often reviewed. ${ }^{3-7}$

Now the framework of polymer solution theory constructed by Flory ${ }^{8,9}$ consists of three concepts: (1) the excluded-volume effect in long flexible (Gaussian) polymer chains, $(2)$ the universality of the unperturbed $(\Theta)$ state without that effect, and (3) the rotational isomeric state (RIS) model for (unperturbed) real chains of arbitrary length. The study of dilute solution behavior of flexible polymers based on the first two concepts was almost completed around 1970, leading to the so-called two-parameter (TP) theory. ${ }^{2}$ It is at almost the same time that the statistical-mechanical method for treating the RIS model, which takes account of the details of the chemical structure and local conformations of the chain on the atomic level, was established by Flory and coworkers. ${ }^{9}$ However, for many equilibrium and steadystate transport problems on stiff or semiflexible polymers, such details are not amenable to mathematical treatments, and moreover are often unnecessary to consider. Some coarse-graining may then be introduced to replace this discrete model by continuous models. In 1976, for this purpose we proposed a general continuous model, called the HW chain. ${ }^{10}$ This new model may describe equilibrium conformational and steady-state transport properties of all kinds of real chains, both flexible and stiff, on the bond length or somewhat longer scales, thus bridging a gap between the RIS model and the classical continuous model, that is, the KratkyPorod (KP) wormlike chain. ${ }^{11}$ Specifically, the transport

\footnotetext{
${ }^{\dagger}$ Professor Emeritus.
}

coefficients were evaluated using the HW cylinder and touched-bead models. Necessarily, these models may be applicable also to short chains or oligomers. In particular, the latter model can give the Einstein intrinsic viscosity for a single bead, although the classical Kirkwood procedure $^{12,13}$ fails to do this. For the study of dynamical properties, the discreteness must be, to some extent, recovered. Thus we devised the dynamic HW chain (with holonomic constraints). ${ }^{14}$ This model enables us to evaluate various dynamical properties, both global and local, in contrast to the Kirkwood (conventional bond) chain. ${ }^{13}$

On the experimental side, since the late 1980s precise measurements have been extended to the oligomer region for a data analysis based on the HW theory. ${ }^{15}$ These have been made possible because well-characterized samples, including oligomers, have become available owing to the progress in polymer synthesis and characterization techniques such as ionic polymerization, GPC, and NMR and also in scattering methods.

All these theoretical and experimental investigations on the basis of the HW model lead to the establishment of a new framework of polymer solution science, ${ }^{16}$ which takes the place of the Flory-Kirkwood framework.

\section{THE HELICAL WORMLIKE CHAIN}

The (unperturbed) HW chain is a general elastic wire model with both bending and torsional energies such that its total elastic (potential) energy becomes a minimum of zero when its contour becomes a regular helix. This helix is called the characteristic helix. Specifically, the model may be described in terms of four basic model parameters: the differential-geometrical curvature $\kappa_{0}$ and torsion $\tau_{0}$ of its characteristic helix, the static stiffness parameter $\lambda^{-1}$, and the shift factor $M_{\mathbf{L}}$ as defined as the molecular weight per unit contour length. The radius $\rho$ and pitch $h$ of the characteristic helix are given by

$$
\begin{aligned}
& \rho=\kappa_{0} /\left(\kappa_{0}^{2}+\tau_{0}^{2}\right) \\
& h=2 \pi \tau_{0} /\left(\kappa_{0}^{2}+\tau_{0}^{2}\right)
\end{aligned}
$$


Thus the model takes account of the stiffness and local conformations of the chain, and may be regarded as a coarse-grained, continuous RIS model. Note that the chain is stiffer for larger $\lambda^{-1}$; the parameter $\lambda$ represents the degree of thermal fluctuation in the chain conformation, and that the total contour length of the chain $L$ is related to its molecular weight $M$ and the number of repeat units in it (or the degree of polymerization) $x$ by

$$
L=M / M_{\mathbf{L}}=\left(M_{0} / M_{\mathbf{L}}\right) x
$$

with $M_{0}$ the molecular weight of the repeat unit. For the details of its statistical mechanics, the reader is referred to Chapter 4 of HWCPS. ${ }^{1}$

The original KP chain ${ }^{11}$ is a special case of the HW chain with $\kappa_{0}=0$ and with vanishing torsional energy, and the chain with $\kappa_{0}=0$ but with the torsional energy is called the generalized KP chain. The characteristic helix becomes a straight line in both cases, as seen from eq 1 . The latter may be classified into two types: type 1 with $\tau_{0} \neq 0$ and type 2 with $\tau_{0}=0$. In this review the original KP chain is referred to simply as the KP chain. Its model parameters are only $\lambda^{-1}$ and $M_{\mathrm{L}}$.

\section{EQUILIBRIUM PROPERTIES}

\section{Mean-Square Radius of Gyration}

We begin by making a comparison of theory with experiment with respect to the dependence on $x$ of the (unperturbed) mean-square radius of gyration $\left\langle S^{2}\right\rangle$ for several flexible and semiflexible polymers to determine their HW or KP model parameters.

Figure 1 shows double-logarithmic plots of $\left\langle S^{2}\right\rangle / x$ (in $\AA^{2}$ ) against $x$ for atactic polystyrene (a-PS) with the fraction of racemic diads $f_{\mathrm{r}}=0.59$ in cyclohexane at $34.5^{\circ} \mathrm{C}(\Theta)^{17,18}$ and in toluene at $15.0^{\circ} \mathrm{C},{ }^{19}$ atactic poly(methyl methacrylate) (a-PMMA) with $f_{\mathrm{r}}=0.79$ in acetonitrile at $44.0^{\circ} \mathrm{C}(\Theta)^{20}$ and in acetone at $25.0^{\circ} \mathrm{C},{ }^{21}$ isotactic (i-) PMMA with $f_{\mathrm{r}}=0.01$ in acetonitrile at $28.0^{\circ} \mathrm{C}(\Theta)^{22}$ and in acetone at $25.0^{\circ} \mathrm{C},{ }^{23} \operatorname{poly}(n$-butyl isocyanate) (PBIC) in tetrahydrofuran (THF) at $40^{\circ} \mathrm{C},{ }^{24}$ and schizophyllan in $0.01 \mathrm{~N} \mathrm{NaOH}$ at $25^{\circ} \mathrm{C} .{ }^{25}$ The data have been obtained from light scattering measurements except for fractions of the flexible polymers with $\left\langle S^{2}\right\rangle^{1 / 2} \lesssim 80 \AA$, for which those have been obtained from small-angle X-ray scattering (SAXS) measurements. In this figure, the solid curves represent the bestfit $\mathrm{HW}$ or KP theoretical values in the $\Theta$ solvents calculated with the values of the model parameters listed in Table I, and the dashed curves connect smoothly the data points for the flexible polymers in the good solvents. In the table are also given the parameter values for polyisobutylene (PIB) in isoamyl isovalerate (IAIV) at $25.0^{\circ} \mathrm{C}(\Theta)^{26}$ and DNA in $0.2 \mathrm{moll}^{-1} \mathrm{NaCl}$ at $25^{\circ} \mathrm{C} .{ }^{27}$ Note that for the above typical semiflexible polymers (PBIC, DNA, and schizophyllan), which may be represented by the KP chain, the excluded-volume effect may be ignored. The data for the flexible polymers in the good solvents in Figure 1 are discussed later.

\section{Chain Stiffness and Local Chain Conformations}

The discussion is limited to the unperturbed chain. In general, the parameter $\lambda^{-1}$ may be considered smaller and larger than about $100 \AA$ for flexible and semiflexible polymers, respectively. This is rather the definition of the chain stiffness from the point of view of the continuous model. It may also be defined by the behavior of the (unperturbed) ratio $\left\langle S^{2}\right\rangle / x$; that is, this ratio becomes independent of $x$ for $x \gtrsim 300$ for flexible polymers but levels off only at much larger $x$ for semiflexible polymers, as seen from Figure 1.

Now the asymptotic ratio $\left(\left\langle S^{2}\right\rangle / x\right)_{\infty}$ for $x=\infty$, which is proportional to the characteristic ratio $C_{\infty},{ }^{9}$ is equal to $8.1_{3}, 6.5_{7}$, and $9.3_{1} \AA^{2}$ for a-PS, a-PMMA, and i-PMMA, respectively, and cannot be directly correlated to the chain stiffness $\lambda^{-1}$, as seen from Table I. The results for $\lambda^{-1}$ in the table indicate that the a-PMMA

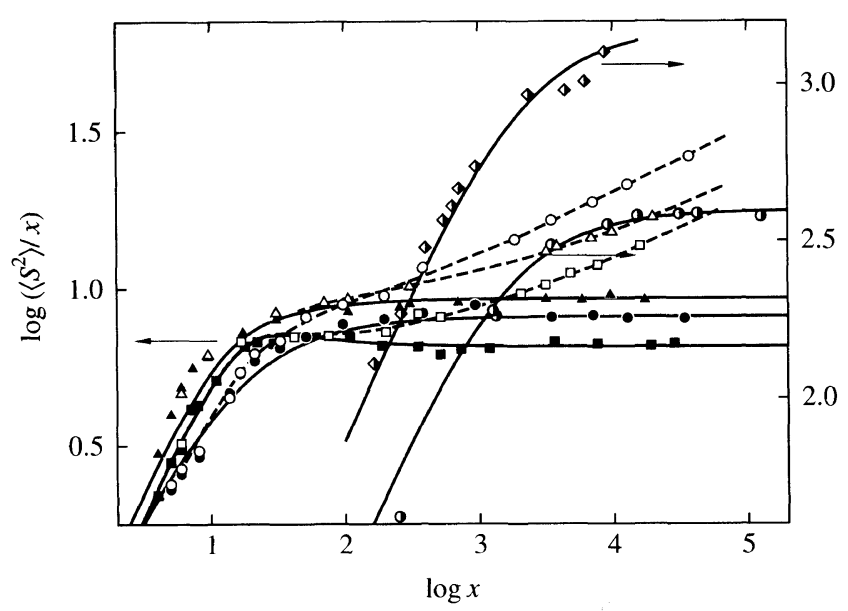

Figure 1. Double-logarithmic plots of $\left\langle S^{2}\right\rangle / x$ (in $\AA^{2}$ ) against $x$ for a-PS in cyclohexane at $34.5^{\circ} \mathrm{C}(\Theta)(\Theta)^{17.18}$ and in toluene at $15.0^{\circ} \mathrm{C}$ (O), ${ }^{19}$ a-PMMA in acetonitrile at $44.0^{\circ} \mathrm{C}(\Theta)(\boldsymbol{\square})^{20}$ and in acetone at $25.0^{\circ} \mathrm{C}(\square),{ }^{21}$ i-PMMA in acetonitrile at $28.0^{\circ} \mathrm{C}(\Theta)(\Delta)^{22}$ and in acetone at $25.0^{\circ} \mathrm{C}(\triangle),{ }^{23} \mathrm{PBIC}$ in $\mathrm{THF}$ at $40^{\circ} \mathrm{C}(\boldsymbol{\bullet}),{ }^{24}$ and schizophyllan in $0.01 \mathrm{~N} \mathrm{NaOH}$ at $25^{\circ} \mathrm{C}(\diamond){ }^{25}$ The solid curves represent the best-fit HW or KP theoretical values, and the dashed curves connect smoothly the data points.

Table I. Values of the HW model parameters for typical flexible and semiflexible polymers

\begin{tabular}{|c|c|c|c|c|c|c|c|}
\hline \multirow{2}{*}{ Polymer $\left(f_{\mathrm{r}}\right)$} & \multirow{2}{*}{ Solvent } & Temp & \multirow{2}{*}{$\lambda^{-1} \kappa_{0}$} & \multirow{2}{*}{$\lambda^{-1} \tau_{0}$} & \multirow{2}{*}{$\frac{\lambda^{-1}}{\AA}$} & \multirow{2}{*}{$\frac{M_{\mathrm{L}}}{\AA^{-1}}$} & \multirow{2}{*}{ ref (Obs.) } \\
\hline & & ${ }^{\circ} \mathrm{C}$ & & & & & \\
\hline a-PS (0.59) & Cyclohexane & 34.5 & 3.0 & 6.0 & 20.6 & 35.8 & 17,18 \\
\hline a-PMMA (0.79) & Acetonitrile & 44.0 & 4.0 & 1.1 & 57.9 & 36.3 & 20 \\
\hline i-PMMA $(0.01)$ & Acetonitrile & 28.0 & 2.5 & 1.3 & 38.0 & 32.5 & 22 \\
\hline PIB & IAIV & 25.0 & 1.0 & 0 & 15.3 & 20.9 & 26 \\
\hline PBIC & THF & 40 & 0 & - & 1320 & 55.1 & 24 \\
\hline DNA & $0.2 \mathrm{moll}^{-1} \mathrm{NaCl}$ & 25 & 0 & - & 1360 & 195 & 27 \\
\hline Schizophyllan & $0.01 N \mathrm{NaOH}$ & 25 & 0 & - & 3000 & 217 & 25 \\
\hline
\end{tabular}




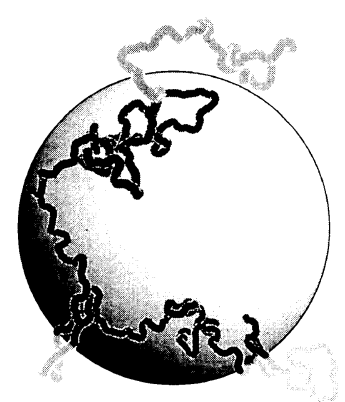

a-PS $\left(f_{\mathrm{r}}=0.59\right)$

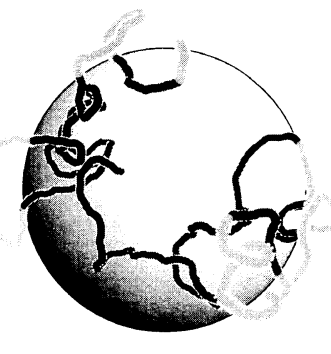

a-PMMA $\left(f_{\mathrm{r}}=0.79\right)$

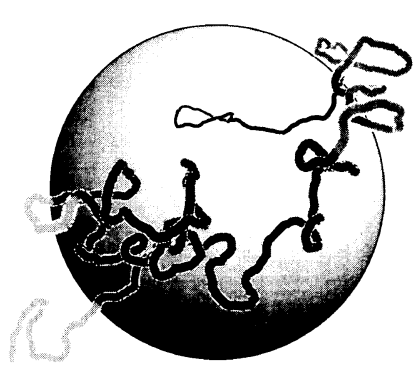

i-PMMA $\left(f_{\mathrm{r}}=0.01\right)$

Figure 2. Representative instantaneous contours of HW Monte Carlo chains (see the text).

chain is much stiffer than the i-PMMA chain, which is stiffer than the a-PS chain, in contrast to the earlier view that the chain is stiffer for larger $C_{\infty}$. Thus $C_{\infty}$ is not a measure of chain stiffness. Further, if $\boldsymbol{R}$ is the end-to-end vector of the continuous chain and if $\boldsymbol{u}_{0}$ is its initial unit tangent vector, the Kuhn segment length $A_{\mathrm{K}}$ and the persistence length $q$ are defined by ${ }^{1}$

$$
\begin{gathered}
A_{\mathrm{K}}=\lim _{L \rightarrow \infty}\left(\left\langle R^{2}\right\rangle / L\right) \\
q=\lim _{L \rightarrow \infty}\left\langle\boldsymbol{R} \cdot \boldsymbol{u}_{0}\right\rangle
\end{gathered}
$$

We then have ${ }^{1}$

$$
A_{\mathrm{K}}=2 q=c_{\infty} \lambda^{-1}=6\left(M_{\mathrm{L}} / M_{0}\right)\left(\left\langle S^{2}\right\rangle / x\right)_{\infty}
$$

with

$$
c_{\infty}=\frac{4+\left(\lambda^{-1} \tau_{0}\right)^{2}}{4+\left(\lambda^{-1} \kappa_{0}\right)^{2}+\left(\lambda^{-1} \tau_{0}\right)^{2}}
$$

Note that $c_{\infty} \leq 1$, where the equality holds only for the $\mathrm{KP}$ chain $\left(\kappa_{0}=0\right)$. Therefore neither $A_{\mathrm{K}}$ nor $q$ is a measure of chain stiffness except for the KP chain.

According to the HW model, a flexible polymer chain in dilute solution may be pictured as a regular helix (the characteristic helix) disturbed (or destroyed) by thermal fluctuations or a random coil retaining more or less helical portions. The regular helical structure is destroyed to a lesser extent in the chain with larger $\lambda^{-1}$. In general, the chain with large $\rho$ (compared to $h$ ) and large $\lambda^{-1}$ is of strong helical nature; that is, it retains rather large and clearly distinguishable helical portions in dilute solution. The chain with vanishing $\rho$ (the KP chain) has no helical nature irrespective of the value of $\lambda^{-1}$ and the chain with small $\lambda^{-1}$ is not of strong helical nature irrespective of the shape of its characteristic helix. We note that the values of $(\rho, h)$ calculated from eq 1 with the parameter values given in Table I are $\left(1.3_{7}, 17_{3}\right),(13.5,23.3)$, and $(12.0,39.1) \AA$ for a-PS, a-PMMA, and i-PMMA, respectively.

In order to visualize the difference in local chain conformation, representative instantaneous contours of $\mathrm{HW}$ Monte Carlo chains are depicted in Figure 2 for a-PS, a-PMMA, and i-PMMA, each with $x=500$, where their radii of gyration $S$ are just equal to the respective values of $\left\langle S^{2}\right\rangle^{1 / 2}$. The shaded sphere has the radius $S$, which is nearly proportional to $\left(\left\langle S^{2}\right\rangle / x\right)_{\infty}^{1 / 2}$. The a-PS chain seems just the random-flight chain. On the other hand, several helical portions are clearly observed in the

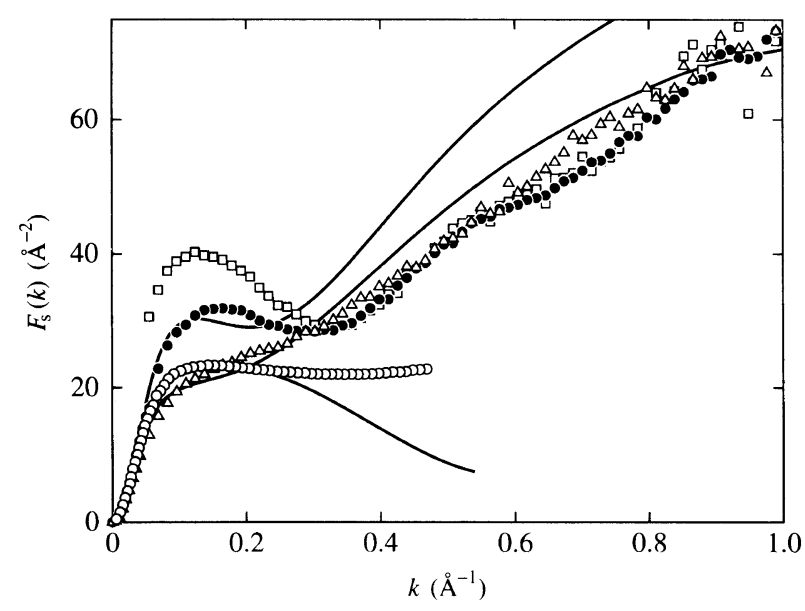

Figure 3. Kratky plots of $F_{\mathrm{s}}(k)$ against $k$ for a-PS $(\bigcirc),{ }^{28}$ a-PMMA (๑), ${ }^{29}$ and i-PMMA $(\triangle),{ }^{30}$ each with $M \simeq 10^{4}$, in the respective $\Theta$ solvents, and s-PMMA $\left(f_{\mathrm{r}}=0.92\right)$ with $M=3.76 \times 10^{4}$ in acetonitrile at $44.0^{\circ} \mathrm{C}(\Theta)(\square){ }^{31}$ The solid curves represent the HW theoretical values for the first three polymers.

picture for a-PMMA, for example, in its bottom-right part, as was expected from the above discussion, while such portions do not appear for i-PMMA. Thus the i-PMMA chain tends to take more extended conformations than the a-PMMA chain, so that the ratio $\left(\left\langle S^{2}\right\rangle / x\right)_{\infty}$ is larger for the former despite the fact that $\lambda^{-1}$ is smaller for the former. However, the chain contour of i-PMMA is still rather smooth compared to that of a-PS. This is due to the fact that $\lambda^{-1}$ is larger for the former than for the latter. It is because of this chain stiffness that the ratio $\left(\left\langle S^{2}\right\rangle / x\right)_{\infty}$ is even larger for i-PMMA than for a-PS. (The ratio is smaller for a-PMMA than for a-PS because of the strong helical nature of the former.) For the chain of strong helical nature such as a-PMMA, $\left\langle S^{2}\right\rangle / x$ exhibits a maximum, as shown in Figure 1.

\section{Scattering Function}

We consider the Kratky function $F_{\mathrm{s}}(k)=M k^{2} P_{\mathrm{s}}(k)$ instead of the scattering function $P_{\mathrm{s}}(k)$ itself, where $k$ is the magnitude of the scattering vector. Figure 3 shows Kratky plots of $F_{\mathrm{s}}(k)$ against $k$ for fractions of a-PS, ${ }^{28}$ a-PMMA, ${ }^{29}$ and $\mathrm{i}-\mathrm{PMMA},{ }^{30}$ each with $M \simeq 10^{4}$, in the respective $\Theta$ solvents. It also includes data for a fraction of syndiotactic (s-) PMMA $\left(f_{\mathrm{r}}=0.92\right)$ with $M=3.76 \times$ $10^{4}$ in acetonitrile at $44.0^{\circ} \mathrm{C}(\Theta) .{ }^{31}$ All the data have been obtained by the use of a point-focussing SAXS camera. 


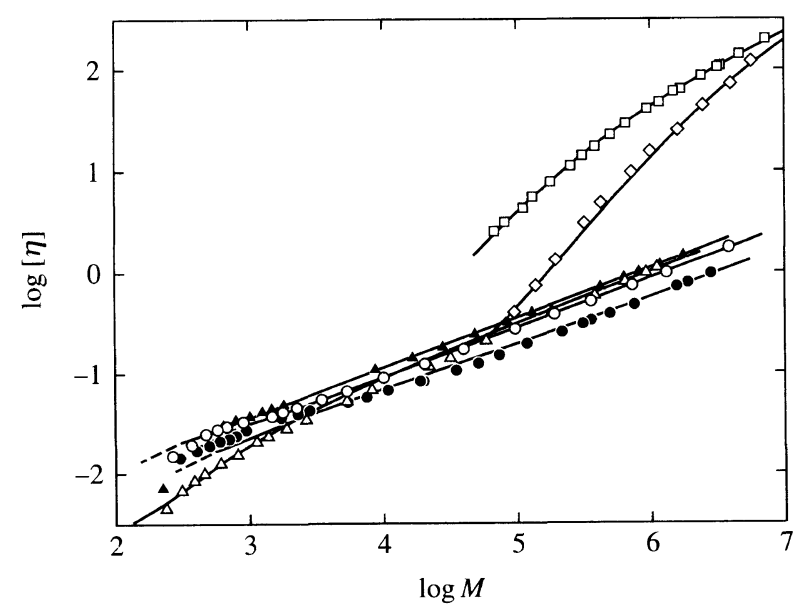

Figure 4. Double-logarithmic plots of $[\eta]$ (in $\mathrm{dl} \mathrm{g}^{-1}$ ) against $M$ for a-PS in cyclohexane at $34.5^{\circ} \mathrm{C}(\Theta)(\bigcirc),{ }^{17,18.41 .42}$ a-PMMA in acetonitrile at $44.0^{\circ} \mathrm{C}(\Theta)(\Theta),{ }^{18,21,43} \mathrm{PIB}$ in IAIV at $25.0^{\circ} \mathrm{C}(\Theta)$ $(\triangle),{ }^{18,44}$ PDMS in MEK at $20.0^{\circ} \mathrm{C}(\Theta)(\triangle),{ }^{45}$ PHIC in $n$-hexane at $25^{\circ} \mathrm{C}(\square),{ }^{46}$ and schizophyllan in water at $25^{\circ} \mathrm{C}(\diamond) .{ }^{47}$ The solid curves represent the best-fit $\mathrm{HW}$ or KP theoretical values, each line segment connecting the $\mathrm{HW}$ values for $N=1$ and 2 .

The solid curves represent the HW theoretical values calculated for the first three polymers by taking account of the spatial distribution of electrons as the scatterers around the chain contour. There is seen to be rather good agreement between theory and experiment.

It is important to see that for a-PMMA (and even for s-PMMA) the observed Kratky plot exhibits the (first) maximum and minimum but not the second ones (or oscillation) such as observed by Kirste and Wunderlich, ${ }^{32,33}$ being consistent with the HW theory prediction. We believe that the desmeared SAXS data obtained by them are not correct for large $k$. We note that the RIS model for a-PMMA can also explain the maximum of $\left\langle S^{2}\right\rangle / x$ at $x \simeq 50$ in Figure 1 but predicts the oscillation in the Kratky plot. ${ }^{34,35}$ This oscillation indicates the "crystal-like" behavior of the RIS chain with discrete internal-rotation angles, and this should be regarded as its defect. Further, we note that the difference in the observed height of the so-called plateau in the Kratky plot between a-PS and a-PMMA cannot be explained by the Gaussian chain model. Although the height is equal to $2 M /\left\langle S^{2}\right\rangle$ for this model, these a-PS and a-PMMA fractions have almost the same $\left\langle S^{2}\right\rangle / M$.

\section{Some Other Topics}

It has been shown that there is rather good agreement between theory and experiment with respect to the dependence on $x$ of the mean-square optical anisotropy $\left\langle\Gamma^{2}\right\rangle$ for a-PS, ${ }^{15,36}$ a-PMMA, ${ }^{36}$ and $\mathrm{i}-\mathrm{PMMA}^{37}$ in the respective $\Theta$ solvents, and also of the mean-square electric dipole moment $\left\langle\mu^{2}\right\rangle$ for a- and i-PMMAs in the respective $\Theta$ solvents $^{38}$ and poly(dimethylsiloxane) (PDMS) in cyclohexane at $25.0^{\circ} \mathrm{C}$. $^{39}$ However, an explanation of $\left\langle\mu^{2}\right\rangle$ for a- and i-PMMAs requires a consideration of possible effects of chain ends since their moments arise from the side groups; this is in contrast to the case of PDMS having rigid perpendicular (type-B) dipoles. We note that the PDMS chain is also of rather strong helical nature and that such a chain has the positive temperature coefficient of $\left\langle R^{2}\right\rangle$ (in the coil limit). ${ }^{40}$

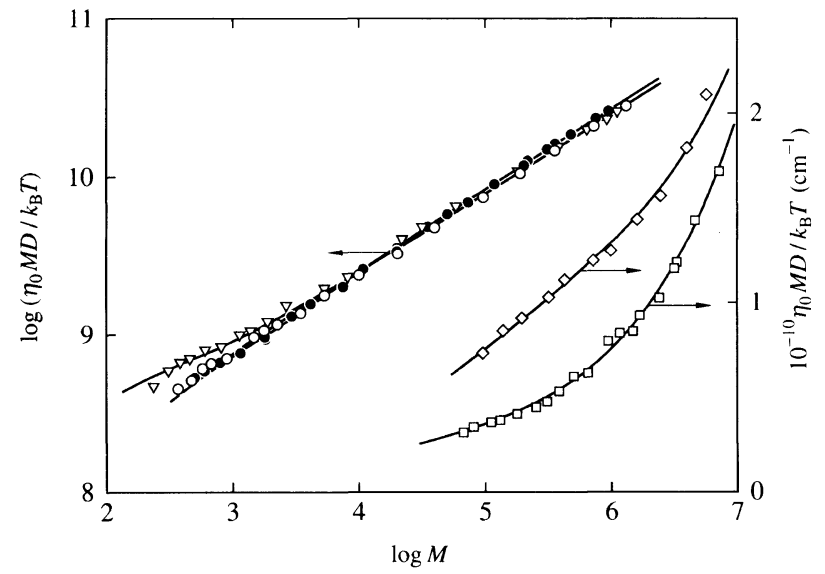

Figure 5. Double-logarithmic plots of $\eta_{0} M D / k_{\mathrm{B}} T$ (in $\mathrm{cm}^{-1}$ ) against $M$ for a-PS in cyclohexane at $34.5^{\circ} \mathrm{C}(\Theta)(\bigcirc),{ }^{18.48}$ a-PMMA in acetonitrile at $44.0^{\circ} \mathrm{C}(\Theta)(\odot),{ }^{18.49,50}$ and PDMS in bromocyclohexane at $29.5^{\circ} \mathrm{C}(\Theta)(\nabla),{ }^{18,45}$ and semi-logarithmic plots of $\eta_{0} M D / k_{\mathrm{B}} T$ against $M$ for PHIC in $n$-hexane at $25^{\circ} \mathrm{C}(\square)^{46}$ and schizophyllan in water at $25^{\circ} \mathrm{C}(\diamond)^{47}$ (see legend for Figure 4).

\section{TRANSPORT PROPERTIES}

\section{HW Cylinder and Touched-Bead Models}

For the evaluation of the steady-state transport coefficients which are valid over a wide range of $M$, including short chains or oligomers, the polymer chain must be treated as a body of finite volume whose surface exerts the frictional force on the solvent fluid. The HW cylinder and touched-bead models are adopted as such polymer hydrodynamic models. Necessarily, the results may be expressed in terms of the cylinder diameter $d$ or the bead diameter $d_{\mathrm{b}}$ in addition to the four basic HW model parameters, as the case may be. Recall that the Kirkwood procedure, ${ }^{12,13}$ which treats the beads as point sources of friction, fails to give the Einstein intrinsic viscosity of the single bead in the extreme the number of them in the chain $N$ is equal to one. For the details, the reader is referred to Chapter 6 of HWCPS. $^{1}$

\section{Intrinsic Viscosity and Translational Diffusion Coefficient}

We consider the intrinsic viscosity $[\eta]$ and the translational diffusion coefficient $D$. In particular, for the HW touched-bead model $[\eta]$ is given by a sum of the Kirkwood-Riseman intrinsic viscosity $[\eta]^{(\mathrm{KR})}$ and the Einstein intrinsic viscosity $[\eta]_{\mathrm{E}}$ of the single bead. Note that $[\eta]^{(\mathrm{KR})}$ vanishes for $N=1$, while $D$ has the Stokes law value for $N=1$. It is convenient to use the HW touched-bead model for flexible polymers, and the KP cylinder model for semiflexible polymers.

Figure 4 shows double-logarithmic plots of $[\eta]$ (in $\mathrm{dl}^{-1}$ ) against $M$ for a-PS in cyclohexane at $34.5^{\circ} \mathrm{C}$ $(\Theta),{ }^{17,18,41,42}$ a-PMMA in acetonitrile at $44.0^{\circ} \mathrm{C}$ $(\Theta),{ }^{18,21,43}$ PIB in IAIV at $25.0^{\circ} \mathrm{C}(\Theta),{ }^{18,44}$ PDMS in methyl ethyl ketone (MEK) at $20.0^{\circ} \mathrm{C}(\Theta),{ }^{45}$ poly $(n-$ hexyl isocyanate) (PHIC) in $n$-hexane at $25^{\circ} \mathrm{C},{ }^{46}$ and schizophyllan in water at $25^{\circ} \mathrm{C} \cdot{ }^{47}$ Figure 5 shows double-logarithmic plots of $\eta_{0} M D / k_{\mathrm{B}} T$ (in $\mathrm{cm}^{-1}$ ) against $M$ for a-PS in cyclohexane at $34.5^{\circ} \mathrm{C}(\Theta),{ }^{18,48}$ a-PMMA in acetonitrile at $44.0^{\circ} \mathrm{C}(\Theta),{ }^{18,49,50}$ and PDMS in bromocyclohexane at $29.5^{\circ} \mathrm{C}(\Theta),{ }^{18,45}$ and semilogarithmic plots of $\eta_{0} M D / k_{\mathrm{B}} T$ against $M$ for PHIC in $n$-hexane at $25^{\circ} \mathrm{C}^{46}$ and schizophyllan in water at $25^{\circ} \mathrm{C},{ }^{47}$ 


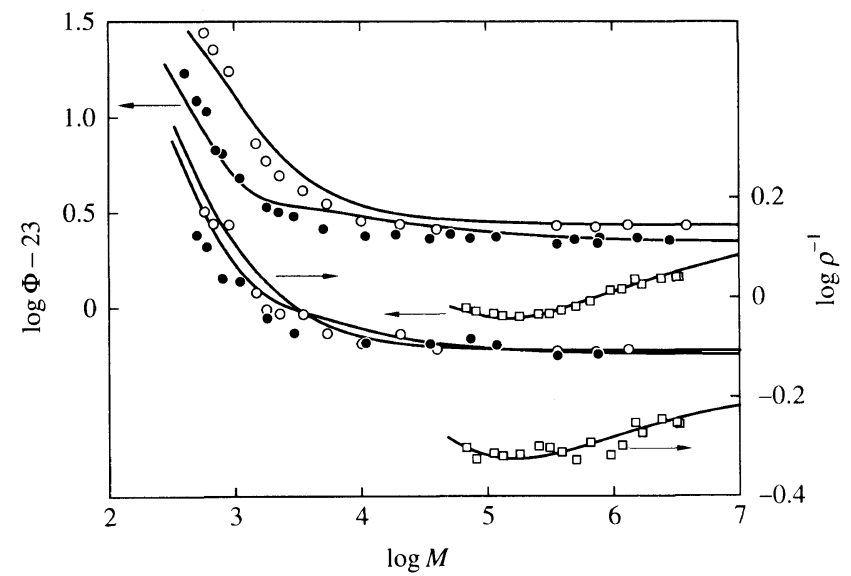

Figure 6. Double-logarithmic plots of $\Phi$ and $\rho^{-1}$ against $M$ for a-PS in cyclohexane at $34.5^{\circ} \mathrm{C}(\Theta)(\bigcirc),{ }^{18,41,48}$ a-PMMA in acetonitrile at $44.0^{\circ} \mathrm{C}(\Theta)(\bigcirc),{ }^{20,43,49}$ and PHIC in $n$-hexane at $25^{\circ} \mathrm{C}(\square){ }^{46}$ The solid curves represent the HW or KP theoretical values (see the text).

where $\eta_{0}$ is the solvent viscosity, $k_{\mathrm{B}}$ the Boltzmann constant, and $T$ the absolute temperature. In Figures 4 and 5, the solid curves represent the best-fit $\mathrm{HW}$ or KP theoretical values, each dashed line segment connecting the HW values for $N=1$ and 2 .

There is seen to be rather good agreement between theory and experiment over a wide range of $M$. In Figure 4 , most important is the fact that for flexible polymers the exponent law for the relation between $[\eta]$ and $M$ holds only in a limited range of $M$, although the exponent becomes asymptotically equal to $1 / 2$ for large $M$. In particular, it is interesting to see that for a-PMMA the double-logarithmic plot of $[\eta]$ against $M$ follows an inverse S-shaped curve, exhibiting the asymptotic behavior only for $M \gtrsim 10^{5}$, as also predicted by the theory. As seen from Figure 5, on the other hand, the deviation of the double-logarithmic plot of $M D$ against $M$ from the asymptotic relation (with slope 1/2) for flexible polymers is rather small, but for a-PMMA the plot clearly follows an S-shaped curve corresponding to the plot of $[\eta]$. Such behavior of $[\eta]$ and $D$ of a-PMMA is characteristic of the chain of strong helical nature.

\section{Some Remarks}

First, we make some remarks on the reduced hydrodynamic volume $\Phi$ and radius $\rho^{-1}$, which may be defined by

$$
\begin{gathered}
\Phi=V_{\mathrm{H}} /\left\langle S^{2}\right\rangle^{3 / 2} \\
\rho^{-1}=R_{\mathrm{H}} /\left\langle S^{2}\right\rangle^{1 / 2}
\end{gathered}
$$

where $V_{\mathrm{H}}=6^{-3 / 2} M[\eta]$ and $R_{\mathrm{H}}=k_{\mathrm{B}} T / 6 \pi \eta_{0} D$ are the hydrodynamic (molar) volume and radius, respectively. Note that $\Phi$ is just the Flory-Fox factor. Figure 6 shows double-logarithmic plots of $\Phi$ and $\rho^{-1}$ against $M$ for a-PS in cyclohexane at $34.5^{\circ} \mathrm{C}(\Theta),{ }^{18,41,48}$ a-PMMA in acetonitrile at $44.0^{\circ} \mathrm{C}(\Theta),{ }^{20,43,49}$ and PHIC in $n$-hexane at $25^{\circ} \mathrm{C} .{ }^{46}$ The solid curves represent the $\mathrm{HW}$ or KP theoretical values, which have been multiplied by the constant ratios of the experimental to theoretical $\Phi_{\infty}$ and $\rho_{\infty}^{-1}$, respectively, for the flexible polymers, where $\Phi_{\infty}$ and $\rho_{\infty}$ are the asymptotic values of $\Phi$ and $\rho$ for large $M$, respectively. It is interesting to see that both theo-

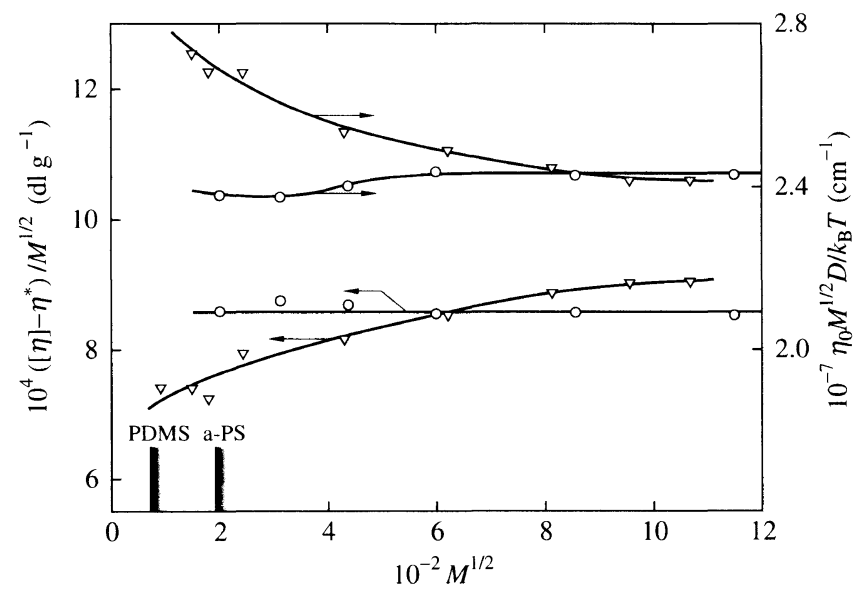

Figure 7. Plots of $\left([\eta]-\eta^{*}\right) / M^{1 / 2}$ and $\eta_{0} M^{1 / 2} D / k_{\mathrm{B}} T$ against $M^{1 / 2}$ for a-PS in cyclohexane at $34.5^{\circ} \mathrm{C}(\Theta)\left(\right.$ with $\left.\eta^{*}=0\right)(\bigcirc)^{17,18.41,42.48}$ and PDMS in bromocyclohexane at $29.5^{\circ} \mathrm{C}(\Theta)$ (with $\eta^{*}=-0.015 \mathrm{dlg}^{-1}$ ) $(\nabla) .{ }^{18,45}$ The solid curves connect the data points smoothly. The HW theoretical values of $\left\langle S^{2}\right\rangle / M$ are almost independent of $M$ to the right of the respective vertical line segments.

retically and experimentally, $\Phi$ and $\rho^{-1}$ increase with decreasing $M$ for small $M$, and in particular, they exhibit a minimum for PHIC. More important is the fact that even in the limit of $M \rightarrow \infty$, the values of $\Phi_{\infty}$ and $\rho_{\infty}^{-1}$ for a-PMMA are definitely different from those for a-PS, indicating that $\Phi$ and $\rho$ are not necessarily universal constants in contrast to the Flory view. Furthermore, the experimental $\Phi_{\infty}$ and $\rho_{\infty}$ for flexible polymers are appreciably smaller than the Kirkwood values ${ }^{2,12,13}$ $2.87 \times 10^{23}$ (exactly $2.862 \times 10^{23}$ ) of $\Phi_{\infty}$ and 1.505 of $\rho_{\infty}$ (even the Zimm value ${ }^{51} 1.479$ of $\rho_{\infty}$ ), respectively, as has often been pointed out. As for the theoretical values, we only note that the $\mathrm{HW}$ theory ${ }^{52}$ with a consideration of fluctuating hydrodynamic interaction gives the values $1.34-1.37$ of $\rho_{\infty}$ (dependent on the system) for flexible polymers and its Zimm value for stiff polymers, which are consistent with experimental results, while it still fails to explain the non-universality of $\Phi_{\infty}$. (For earlier theoretical investigations, see Chapter 6 of HWCPS. ${ }^{1}$ )

Second, we note that the values of $[\eta]$ of PIB and PDMS in the oligomer region become negative, and therefore have not been plotted in Figure 4. In this case $[\eta]$ may be written in the form, ${ }^{44}$

$$
[\eta]=[\eta]^{(\mathrm{HW})}+\eta^{*}
$$

where $[\eta]^{(\mathrm{HW})}$ is a sum of $[\eta]^{(\mathrm{KR})}$ and $[\eta]_{\mathrm{E}}$, and $\eta^{*}$ is an empirical additional nonpositive parameter independent of $M$. The negative $[\eta]$ may be regarded as arising from specific interactions between solute and solvent molecules such that a liquid structure of some kind existing in the solvent is destroyed in the vicinity of a solute molecule.

The final remark is concerned with the so-called "draining effect." Figure 7 shows plots of $\left([\eta]-\eta^{*}\right) / M^{1 / 2}$ and $\eta_{0} M^{1 / 2} D / k_{\mathrm{B}} T$ against $M^{1 / 2}$ for a-PS in cyclohexane at $34.5^{\circ} \mathrm{C}(\Theta)^{17,18,41,42,48}$ and PDMS in bromocyclohexane at $29.5^{\circ} \mathrm{C}(\Theta) .{ }^{18,45}$ The solid curves connect the data points smoothly, and the vertical line segments with shadows indicate the values of $M$ above which the HW theoretical values of $\left\langle S^{2}\right\rangle / M$ become almost independent of $M$ for the respective polymers. It is seen that for PDMS $\left([\eta]-\eta^{*}\right) / M^{1 / 2}$ decreases and $M^{1 / 2} D$ increases with decreasing $M$ even in the range of $M$ where the (unper- 


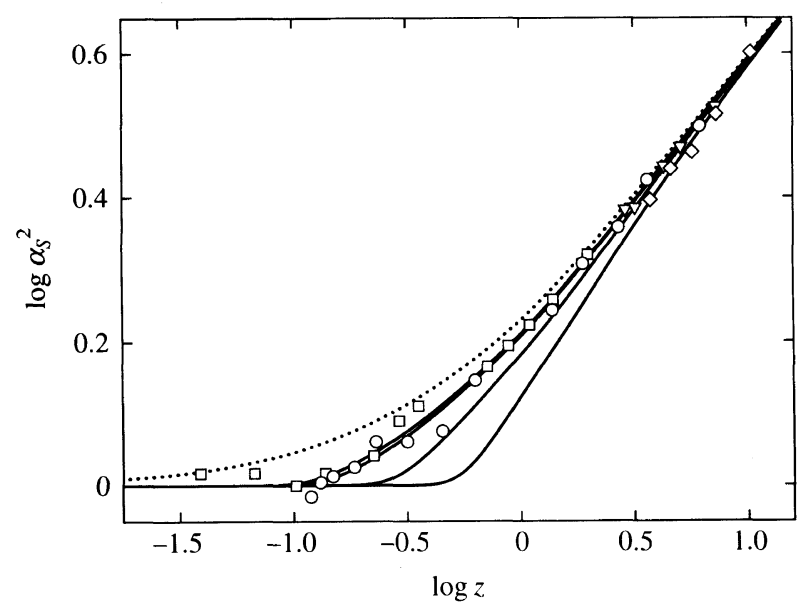

Figure 8. Double-logarithmic plots of $\alpha_{S}^{2}$ against $z$ for a-PS in toluene at $15.0^{\circ} \mathrm{C}(\bigcirc),{ }^{19}$ a-PMMA in acetone at $25.0^{\circ} \mathrm{C}(\square)$ and in chloroform at $25.0^{\circ} \mathrm{C}(\diamond),{ }^{21}$ and i-PMMA in chloroform at $25.0^{\circ} \mathrm{C}(\nabla) \cdot{ }^{23}$ The solid and dotted curves represent the QTP and TP theory values, respectively.

turbed) static properties such as $\left\langle S^{2}\right\rangle$ exhibit the Gaussian chain behavior, in contrast to the case of a-PS. This indicates that the effect exists for PDMS. An analysis of such data by the use of the HW theory leads to remarkably small values of $d_{\mathrm{b}}$, suggesting that the nonslip boundary condition on the bead surface may break down.

\section{EXCLUDED-VOLUME EFFECTS}

\section{Gyration-Radius Expansion Factor}

In order to treat the excluded-volume effect, we consider the HW chain on which $n+1$ beads are arrayed with spacing $a$ between them along the contour, so that $L=n a$, and suppose that there exist excluded-volume interactions between them expressed in terms of the usual binary-cluster integral $\beta .^{2}$

In this subsection we consider the gyration-radius expansion factor $\alpha_{S}$ defined by

$$
\left\langle S^{2}\right\rangle=\left\langle S^{2}\right\rangle_{0} \alpha_{S}^{2}
$$

Here and hereafter, the subscript 0 indicates the unperturbed value. The TP theory ${ }^{2}$ predicts that $\alpha_{S}$ is a function only of the conventional excluded-volume parameter $z$. For the perturbed HW chain, however, $\alpha_{S}$ is a function only of the intramolecular scaled excludedvolume parameter $\tilde{z}$; that is, ${ }^{53-55}$

$$
\alpha_{S}=\alpha_{S}(\tilde{z})
$$

with

$$
\tilde{z}=(3 / 4) K(\lambda L) z
$$

where the function $K$ of $\lambda L$ represents the effect of chain stiffness. As $\lambda L$ is increased from 0 to $\infty, K$ increases from 0 to $4 / 3$, so that $z$ approaches $z$. The parameter $z$ is now defined by

$$
z=(3 / 2 \pi)^{3 / 2}(\lambda B)(\lambda L)^{1 / 2}
$$

with $B$ the excluded-volume strength given by

$$
B=\beta / a^{2} c_{\infty}^{3 / 2}
$$

where $a=M_{0} / M_{\mathrm{L}}$ if the repeat unit is taken as a single

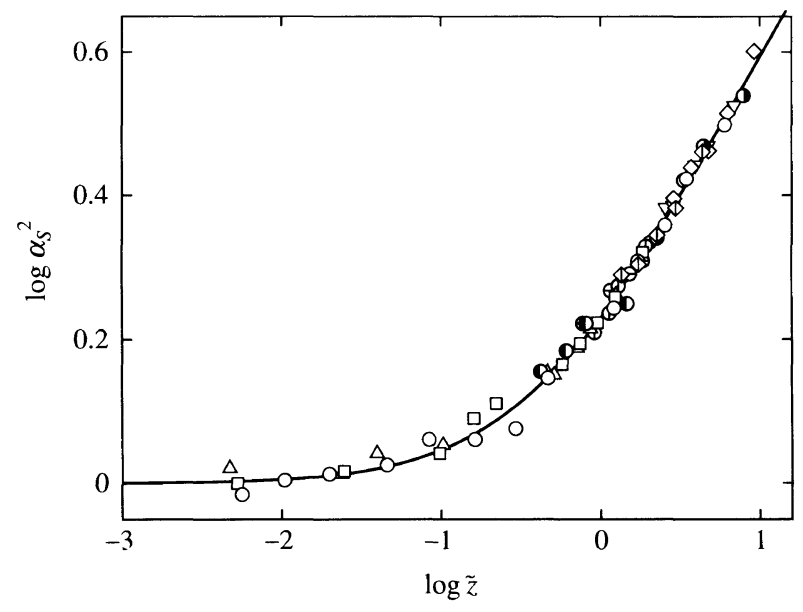

Figure 9. Double-logarithmic plots of $\alpha_{S}^{2}$ against $\tilde{z}$ with the same data as those in Figure 8 along with those for a-PS in benzene at $25.0^{\circ} \mathrm{C}$ (O) and in MEK at $35.0^{\circ} \mathrm{C}(\mathrm{D}),{ }^{57} \mathrm{a}-\mathrm{PMMA}$ in nitroethane at $30.0^{\circ} \mathrm{C}$ $(\diamond),{ }^{21} \mathrm{i}$-PMMA in acetone at $25.0^{\circ} \mathrm{C}(\triangle),{ }^{23} \mathrm{PIB}$ in $n$-heptane at $25.0^{\circ} \mathrm{C}$ ( $),{ }^{26,42}$ and PDMS in toluene at $25.0^{\circ} \mathrm{C}(\ominus) .{ }^{58}$ The solid curve represents the QTP theory values.

bead, and $c_{\infty}$ is given by eq 6 . The function $\alpha_{S}(\tilde{z})$ may be obtained by replacing $z$ by $\tilde{z}$ in a TP expression for $\alpha_{S}(z)$. This is the quasi-two-parameter (QTP) scheme. It is then convenient to adopt the Domb-Barrett expression for $\alpha_{S}{ }^{56}$

Now $\left\langle S^{2}\right\rangle_{0}$ in a given good solvent cannot be directly determined since it may in general depend on solvent and temperature. However, the (intramolecular) excluded-volume effect must disappear in the oligomer region, so that we have $\left\langle S^{2}\right\rangle=\left\langle S^{2}\right\rangle_{0}$ there. Therefore, if we choose the solvent and temperature so that in the oligomer region $\left\langle S^{2}\right\rangle$ coincides with the unperturbed mean-square radius of gyration in a proper $\Theta$ solvent (at $T=\Theta$ ), which we denote by $\left\langle S^{2}\right\rangle_{\Theta}$, then the latter may be regarded as equal to $\left\langle S^{2}\right\rangle_{0}$ in that good solvent for all values of $M$; that is,

$$
\left\langle S^{2}\right\rangle_{0}=\left\langle S^{2}\right\rangle_{\Theta}
$$

Taking this $\Theta$ solvent as a reference standard, we may then determine $\alpha_{S}$ from $\alpha_{S}^{2}=\left\langle S^{2}\right\rangle /\left\langle S^{2}\right\rangle_{\Theta}$. It is seen that the good-solvent systems in Figure 1 fulfil the requirement of eq 15 .

Figure 8 shows double-logarithmic plots of $\alpha_{S}^{2}$ against $z$ for a-PS in toluene at $15.0^{\circ} \mathrm{C},{ }^{19}$ a-PMMA in acetone at $25.0^{\circ} \mathrm{C}$ and in chloroform at $25.0^{\circ} \mathrm{C},{ }^{21}$ and i-PMMA in chloroform at $25.0^{\circ} \mathrm{C}^{23}$ The solid and dotted curves represent the QTP and TP theory values, respectively. There is good agreement between the QTP theoretical and experimental values. The solid curves (or data points) do not form a single-composite curve but deviate downward progressively from the dotted curve with decreasing $z$ (or $M$ ) because of the effect of chain stiffness. The effect is larger for better solvents (with larger $\lambda B$ ). It is surprising to see that the effect on $\alpha_{S}$ remains rather large even at $z \simeq 10$ or at $M \simeq 10^{6}$. Figure 9 shows double-logarithmic plots of $\alpha_{S}^{2}$ against $\tilde{z}$ with the same data as those in Figure 8 along with those for a-PS in benzene at $25.0^{\circ} \mathrm{C}$ and in $\mathrm{MEK}$ at $35.0^{\circ} \mathrm{C},{ }^{57}$ a-PMMA in nitroethane at $30.0^{\circ} \mathrm{C},{ }^{21}$ i-PMMA in acetone at $25.0^{\circ} \mathrm{C},{ }^{23} \mathrm{PIB}$ in $n$-heptane at $25.0^{\circ} \mathrm{C},{ }^{26,42}$ and PDMS in toluene at $25.0^{\circ} \mathrm{C} .{ }^{58}$ The solid curve represents the 


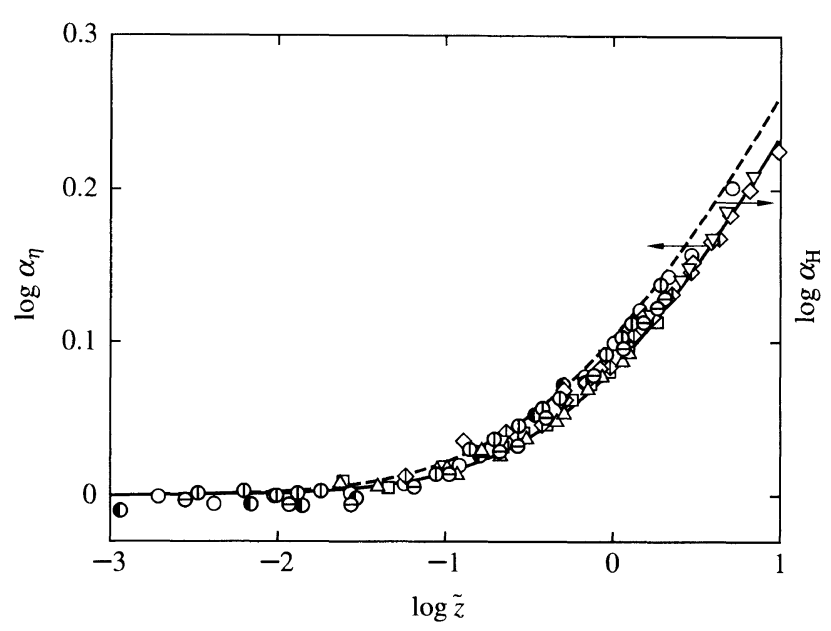

Figure 10. Double-logarithmic plots of $\alpha_{\eta}$ against $\tilde{z}$ with the data for the same systems as those in Figure 9. The solid and dashed curves represent the QTP theory values of $\alpha_{\eta}$ and $\alpha_{\mathrm{H}}$, respectively, calculated from the Barrett equations ${ }^{59}$ with $\tilde{z}$ in place of $z$.

QTP theory values. It is seen that all the data points form a single-composite curve and are fitted by the solid curve irrespective of the differences in polymer species (chain stiffness and local chain conformation) and solvent condition, indicating that the QTP scheme is valid for $\alpha_{s}$.

Viscosity- and Hydrodynamic-Radius Expansion Factors

The viscosity- and hydrodynamic-radius expansion factors $\alpha_{\eta}$ and $\alpha_{\mathrm{H}}$ are defined by

$$
\begin{gathered}
{[\eta]=[\eta]_{0} \alpha_{\eta}^{3}} \\
R_{\mathrm{H}}=R_{\mathrm{H}, 0} \alpha_{\mathrm{H}}
\end{gathered}
$$

Even if eq 15 holds, there are several difficulties in the experimental determination of $\alpha_{\eta}$ and $\alpha_{H}$ because of the solvent dependence of $\Phi_{\Theta}$ and also because of the specific interaction $\eta^{*}$ and/or the solvent dependence of $d_{\mathrm{b}}$. For the details, the reader is referred to Chapter 8 of HWCPS. ${ }^{1}$

Figure 10 shows double-logarithmic plots of $\alpha_{\eta}$ against $\tilde{z}$ for a-PS in toluene at $15.0^{\circ} \mathrm{C},{ }^{42}$ in benzene at $25.0^{\circ} \mathrm{C},{ }^{57}$ and in MEK at $35.0^{\circ} \mathrm{C},{ }^{57}$ a-PMMA in acetone at $25.0^{\circ} \mathrm{C}$, in chloroform at $25.0^{\circ} \mathrm{C}$, and in nitroethane at $30.0^{\circ} \mathrm{C},{ }^{21}$ i-PMMA in acetone at $25.0^{\circ} \mathrm{C}$ and in chloroform at $25.0^{\circ} \mathrm{C},{ }^{23} \mathrm{PIB}$ in $n$-heptane at $25.0^{\circ} \mathrm{C},{ }^{42}$ and PDMS in toluene at $25.0^{\circ} \mathrm{C} .{ }^{58}$ The data points for $\alpha_{\mathrm{H}}$, which have not been plotted in Figure 10, coincide with those for $\alpha_{\eta}$ within experimental error. (This is surprising.) The solid and dashed curves represent the QTP theory values of $\alpha_{\eta}$ and $\alpha_{\mathrm{H}}$, respectively, calculated from the Barrett equations $^{59}$ with $\tilde{z}$ in place of $z$. Thus there is good agreement between theory and experiment for $\alpha_{\eta}$ but this is not the case with $\alpha_{H}$. This disagreement for $\alpha_{H}$ may be regarded as arising from the effect of fluctuating hydrodynamic interaction. ${ }^{60,61}$ However, it may be concluded that the QTP scheme is valid for both $\alpha_{\eta}$ and $\alpha_{H}$ as well as for $\alpha_{S}$.

\section{Second Virial Coefficient}

We assume that the two end beads are different from the $n-1$ intermediate ones and also from each other in

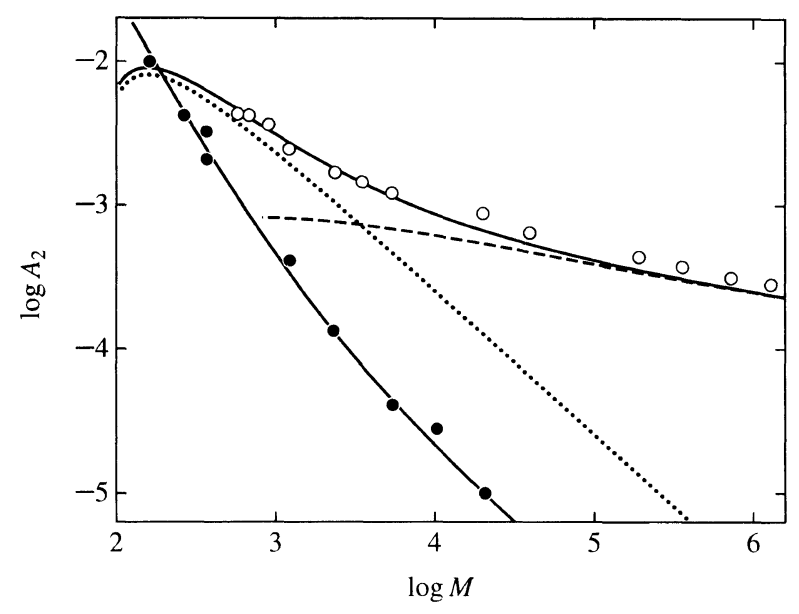

Figure 11. Double-logarithmic plots of $A_{2}$ (in $\left.\mathrm{cm}^{3} \mathrm{~mol} \mathrm{~g}^{-2}\right)$ against $M$ for a-PS in cyclohexane at $34.5^{\circ} \mathrm{C}(\Theta)(\bigcirc)$ and in toluene at $15.0^{\circ} \mathrm{C}$ (O).$^{63}$ The solid curves represent the theoretical values, and the dashed and dotted curves those of $A_{2}^{(\mathrm{HW})}$ and $A_{2}^{(\mathrm{E})}$ for the latter system, respectively.

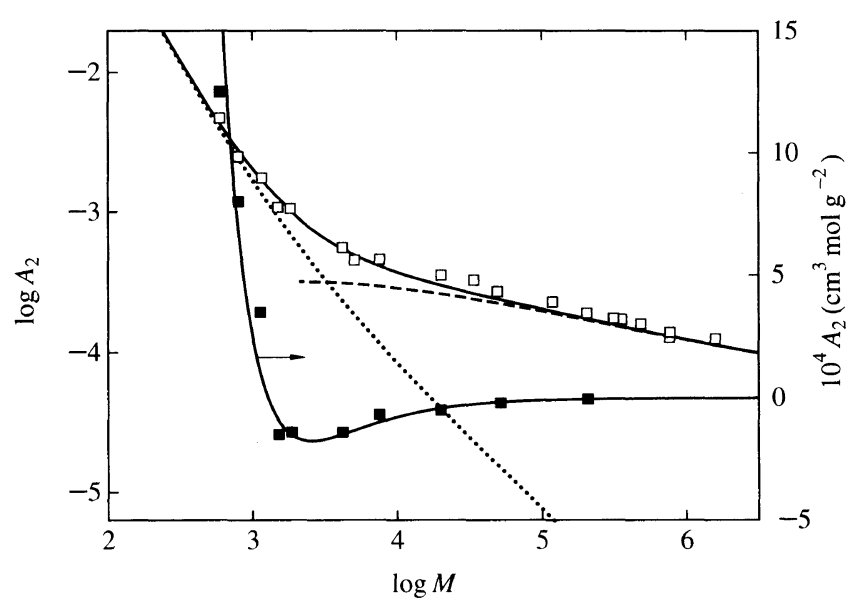

Figure 12. Double- and semi-logarithmic plots of $A_{2}$ against $M$ for a-PMMA in acetonitrile at $44.0^{\circ} \mathrm{C}(\Theta)(\square)$ and in acetone at $25.0^{\circ} \mathrm{C}$ ( $\square){ }^{69}$ The curves have the same meaning as those in Figure 11.

species. According to the HW theory, ${ }^{62}$ the second virial coefficient $A_{2}$ may then be written in the form,

$$
A_{2}=A_{2}^{(\mathrm{HW})}+A_{2}^{(\mathrm{E})}
$$

where $A_{2}^{(\mathrm{E})}$ represents the contribution of possible effects of chain ends to $A_{2}$, and $A_{2}^{(\mathrm{HW})}$ is the part of $A_{2}$ without those effects, or $A_{2}$ for the (fictitious) chain composed of $n+1$ identical beads.

The first term $A_{2}^{(\mathrm{HW})}$ may be written in the form,

$$
A_{2}^{(\mathrm{HW})}=4 \pi^{3 / 2} N_{\mathrm{A}}\left(\left\langle S^{2}\right\rangle^{3 / 2} / M^{2}\right) \Psi
$$

where $N_{\mathrm{A}}$ is the Avogadro constant and $\Psi$ is the interpenetration function given by

$$
\Psi=\left(6 \lambda\left\langle S^{2}\right\rangle_{0} / c_{\infty} L\right)^{-3 / 2}\left(z / \alpha_{S}^{3}\right) h(\tilde{z}, \tilde{z})
$$

In eq $20, \tilde{\tilde{z}}$ is the intermolecular scaled excluded-volume parameter defined by

$$
\tilde{z}=[Q(\lambda L) / 2.865] z
$$

where the function $Q$ of $\lambda L$ also represents the effect of chain stiffness and becomes equal to 2.865 in the limit of $\lambda L \rightarrow \infty$. In this limit, therefore, $\tilde{z}$ also approaches $z$, and the function $h$ becomes the so-called $h$ function in 


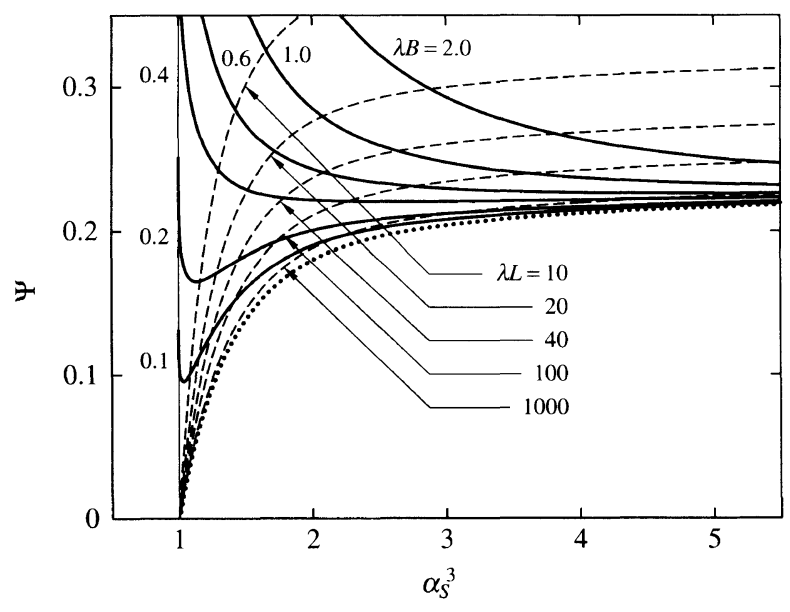

Figure 13. Plots of the theoretical $\Psi$ against $\alpha_{S}^{3}$ for a-PS. The solid and dashed curves represent the values at constant $\lambda B$ and $\lambda L$, respectively. The dotted curve represents the TP theory values.

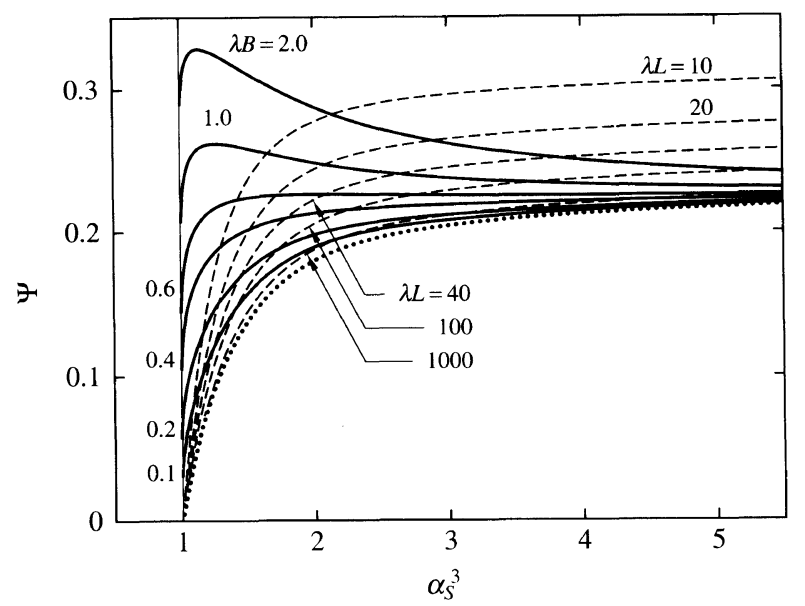

Figure 14. Plots of the theoretical $\Psi$ against $\alpha_{S}^{3}$ for a-PMMA. The curves have the same meaning as those in Figure 13.

the TP theory. ${ }^{2}$ Thus, in general, neither the TP nor the QTP scheme is valid for $A_{2}^{(\mathrm{HW})}$. The second term $A_{2}^{(\mathrm{E})}$ may be written in the form,

$$
A_{2}^{(\mathrm{E})}=a_{1} M^{-1}+a_{2} M^{-2}
$$

where the coefficients $a_{1}$ and $a_{2}$ involve the binary-cluster integrals associated with the end beads but are independent of $M$, so that $A_{2}^{(\mathrm{E})}$ vanishes for large $M$.

At the $\Theta$ temperature, which is now defined as the temperature at which $A_{2}$ vanishes for large $M, A_{2}^{(\mathrm{HW})}$ and $\beta$ must vanish, so that $A_{2}$ at the $\Theta$ temperature, which we denote by $A_{2, \Theta}$, is given by

$$
A_{2, \Theta}=A_{2}^{(\mathrm{E})}
$$

This indicates that $A_{2, \Theta}$ does not vanish except at large $M$. Thus the TP scheme is valid also for $A_{2}$ only in the limit of $M \rightarrow \infty$.

Figure 11 shows double-logarithmic plots of $A_{2}$ (in $\mathrm{cm}^{3} \mathrm{molg}^{-2}$ ) against $M$ for a-PS in cyclohexane at $34.5^{\circ} \mathrm{C}(\Theta)$ and in toluene at $15.0^{\circ} \mathrm{C} .{ }^{63}$ The solid curves represent the theoretical values, and the contributions of $A_{2}^{(\mathrm{HW})}$ and $A_{2}^{(\mathrm{E})}$ for the latter system are shown by the dashed and dotted curves, respectively. It is seen that there is rather good agreement between theory and experiment, and that $A_{2}^{(\mathrm{E})}$ remains appreciable up to

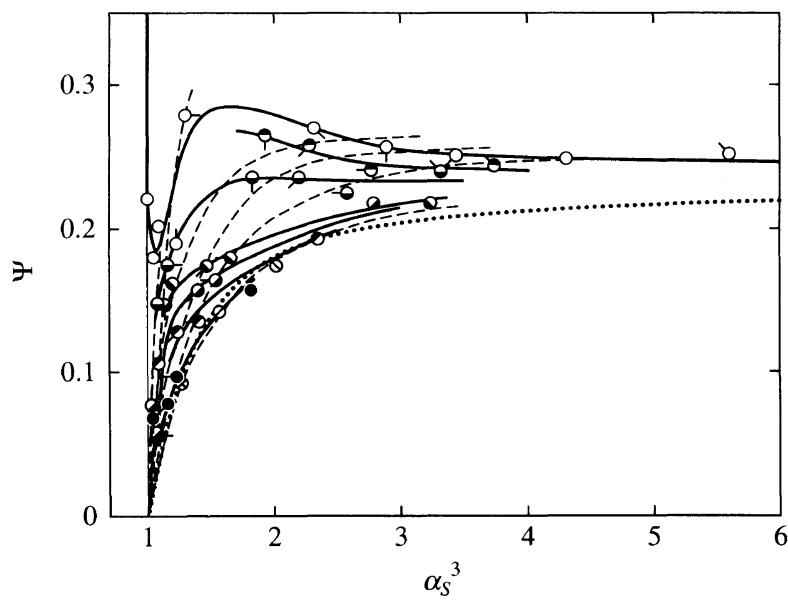

Figure 15. Plots of $\Psi$ against $\alpha_{S}^{3}$ for a-PS; $\bigcirc$, in toluene at $15.0^{\circ} \mathrm{C}$; $\Theta$, in $n$-butyl chloride at $15.0^{\circ} \mathrm{C} ; \Theta$, in 4 - $t$-butyltoluene at $50.0^{\circ} \mathrm{C} ; \boldsymbol{\Theta}$, in cyclohexane $(\mathrm{CH})$ at $55.0^{\circ} \mathrm{C} ; \boldsymbol{O}$, in $\mathrm{CH}$ at $50.0^{\circ} \mathrm{C} ; \boldsymbol{O}$, in $\mathrm{CH}$ at $45.0^{\circ} \mathrm{C}$;, in $\mathrm{CH}$ at $40.0^{\circ} \mathrm{C}$; $\ominus$, in $\mathrm{CH}$ at $42.0^{\circ} \mathrm{C}$; $\oslash$, in $\mathrm{CH}$ at $38.0^{\circ} \mathrm{C}$; $\otimes$, in $\mathrm{CH}$ at $36.0^{\circ} \mathrm{C} .^{64-66}$ Various directions of pips indicate different values of $M$. The solid and dashed curves connect smoothly the data points at constant $B$ and $M$, respectively. The dotted curve represents the TP theory values.

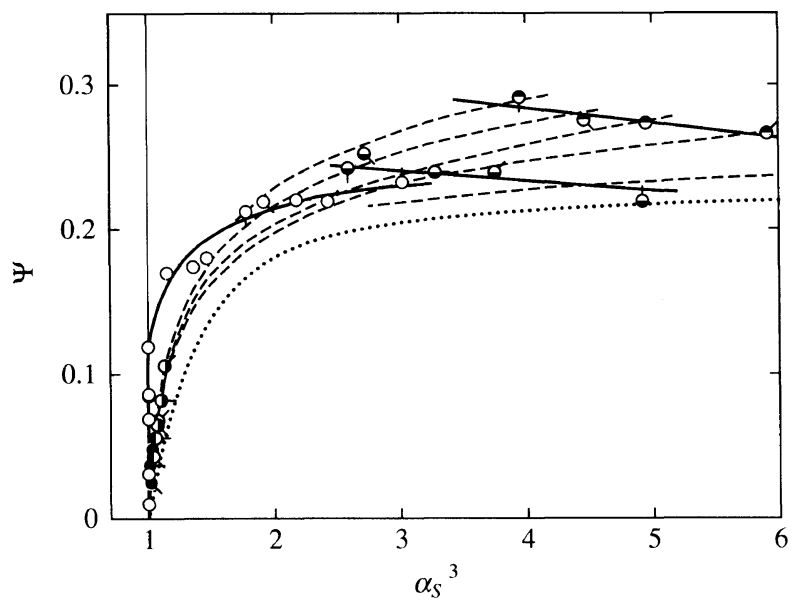

Figure 16. Plots of $\Psi$ against $\alpha_{S}^{3}$ for a-PMMA; ${ }^{64} \bigcirc$, in acetone at $25.0^{\circ} \mathrm{C}$; $\Theta$, in chloroform at $25.0^{\circ} \mathrm{C}$; $\Theta$, in nitroethane at $30.0^{\circ} \mathrm{C} ; \boldsymbol{O}$, in acetonitrile $(\mathrm{AN})$ at $55.0^{\circ} \mathrm{C} ; \mathrm{D}$, in $\mathrm{AN}$ at $50.0^{\circ} \mathrm{C}$; $\mathrm{O}$, in $\mathrm{AN}$ at $47.0^{\circ} \mathrm{C}$ (see legend for Figure 15).

$M=10^{4}-10^{5}$. Figure 12 shows similar plots for a-PMMA in acetonitrile at $44.0^{\circ} \mathrm{C}(\Theta)$ and in acetone at $25.0^{\circ} \mathrm{C} .{ }^{64}$ The curves have the same meaning as those in Figure 11. It is interesting to see that $A_{2, \Theta}$ for a-PMMA exhibits a minimum.

Now we examine the behavior of $\Psi$. Its theoretical values as a function of $\alpha_{S}^{3}$ are plotted in Figures 13 and 14 for a-PS and a-PMMA, respectively. The dotted curves represent the TP theory values. The solid curves represent the values for the case in which $\lambda L$ (or $M$ ) is changed at constant $\lambda B$, while the dashed curves represent the values for the case in which $\lambda B$ is changed at constant $\lambda L$ (or $M$ ). It is seen that the TP theory prediction is obtained as the asymptotic limit of $\lambda L \rightarrow \infty$ or $\lambda B \rightarrow 0$, that for finite $\lambda L$ and $\lambda B, \Psi$ always deviates upward from the TP theory prediction, and that the behavior of $\Psi$ depends remarkably on chain stiffness and local chain conformation.

Figures 15 and 16 show plots of $\Psi$ against $\alpha_{S}^{3}$ for

Polym. J., Vol. 31, No. 2, 1999 
a-PS ${ }^{64-66}$ and a-PMMA, ${ }^{64}$ respectively, where various types of circles indicate different solvent conditions (different $B$ ), and various directions of pips attached to them indicate different values of $M$. The solid and dashed curves connect smoothly the data points at constant $B$ and $M$, respectively. There is semiquantitative agreement between theory and experiment, as seen from a comparison of Figures 15 and 16 with Figures 13 and 14 , respectively. In particular, it is interesting to see that as $\alpha_{S}^{3}$ (or $M$ ) is decreased in their respective good solvents of almost the same $\lambda B$, toluene $(\lambda B=0.26)$ and acetone $(\lambda B=0.22)$, for a-PS $\Psi$ increases steeply (at $\alpha_{S}=1$ ) after passing through a maximum and then a minimum at $\alpha_{S}^{3} \simeq 1$, while for a-PMMA it decreases monotonically and then drops suddenly after reaching a finite value at $\alpha_{S}=1$

\section{Some Remarks}

We make some remarks on the behavior of $\alpha_{S}$ and $A_{2}$ below $\Theta$. With experimental values of $\beta$ correctly determined, it has been found that the QTP scheme is valid for $\alpha_{S}$ also below $\Theta$, while the TP scheme is valid for $A_{2}^{(\mathrm{HW})}$ below $\Theta$ in contrast to its behavior above $\Theta .^{67-70}$ In particular, we note that $\beta$ is not proportional to $1-\Theta / T$ for a-PS below $\Theta{ }^{68,70}$ As for the third virial coefficient $A_{3}$, we only note that it may be given by a sum of terms explicitly involving the ternary-cluster integral $\beta_{3}$, a term $A_{3}^{(\mathrm{HW})}$ involving only (effective) $\beta$, and a term $A_{3}^{(\mathrm{E})}$ representing effects of chain ends, so that it does not vanish at $\Theta$ even for large $M .^{71-73}$

\section{DYNAMICAL PROPERTIES}

\section{Dynamic HW Chains}

We consider a chain composed of $N$ identical rigid subbodies (beads), not necessarily spherical, joined successively with bonds of fixed length $a$, where their centers are located nearly on the contour of the continuous HW chain of length $L$ and each subbody has translational and rotatory friction constants $\zeta_{t}$ and $\zeta_{r}$. The bond length $a$ is not equal to the spacing $a$ introduced in the last section. For flexible chains one subbody as a motional unit may be regarded as corresponding to two successive skeletal bonds of the real chain, that is, the repeat unit, so that $L / N=M_{0} / M_{\mathrm{L}}$. This is the dynamic $\mathrm{HW}$ chain. ${ }^{14}$ In the preaveraging approximation to its constraining matrix, for this constrained chain we can derive two types of diffusion equations which are suitable for the treatments of global and local motions, respectively. ${ }^{74}$ Recall that in the same approximation the Kirkwood or Fixman-Kovac conventional bond chain ${ }^{75}$ fails to treat local motions since the associated eigenvalues become negative. In the following we discuss only two recent topics on dynamic light scattering. For the details of the chain dynamics and also classical problems such as dielectric and magnetic relaxation, the reader is referred to Chapters 9 and 10 of HWCPS. ${ }^{1}$

\section{Dynamic Depolarized Light Scattering}

Let $J_{\Gamma}(\Delta \omega)$ be the depolarized component of the dynamic light scattering intensity such that it gives the mean-square optical anisotropy $\left\langle\Gamma^{2}\right\rangle$ when integrated over the difference $\Delta \omega$ between the angular frequencies

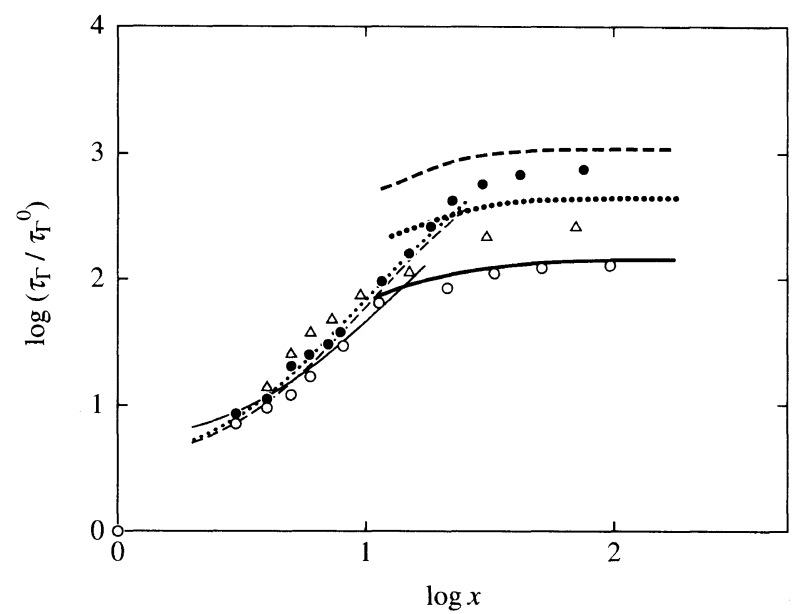

Figure 17. Double-logarithmic plots of $\tau_{\Gamma} / \tau_{\Gamma}^{0}$ against $x$ for a-PS in cyclohexane at $34.5^{\circ} \mathrm{C}(\Theta)(O),{ }^{76}$ a-PMMA in acetonitrile at $44.0^{\circ} \mathrm{C}$ $(\Theta)(\odot),{ }^{77}$ and $i-P M M A$ in acetonitrile at $28.0^{\circ} \mathrm{C}(\Theta)(\triangle) .{ }^{78}$ The heavy solid, dashed, and dotted curves represent the respective $\mathrm{HW}$ theoretical values, and the light curves represent the respective theoretical values for the rigid sphere model.

of the scattered and incident light waves at vanishing magnitude $k$ of the scattering vector. We then define a depolarized scattering correlation time $\tau_{\Gamma}$ as the reciprocal of the half-width at half-maximum of the spectrum $J_{\Gamma}$. The ratio $\tau_{\Gamma} / \tau_{\Gamma}^{0}$ of $\tau_{\Gamma}$ to its value $\tau_{\Gamma}^{0}$ for the monomer in the limit of $x \rightarrow \infty$ may be regarded as a measure of dynamic chain stiffness. (Such measures may be defined also for dielectric and magnetic correlation times.)

Figure 17 shows double-logarithmic plots of $\tau_{\Gamma} / \tau_{\Gamma}^{0}$ against $x$ for a-PS in cyclohexane at $34.5^{\circ} \mathrm{C}(\Theta),{ }^{76}$ a-PMMA in acetonitrile at $44.0^{\circ} \mathrm{C}(\Theta),{ }^{77}$ and i-PMMA in acetonitrile at $28.0^{\circ} \mathrm{C}(\Theta) .^{78}$ The heavy solid, dashed, and dotted curves represent the respective $\mathrm{HW}$ theoretical values. They cannot be obtained in the oligomer region because of the (block-diagonal) approximation used, and therefore we have calculated the theoretical values there using simply the rigid sphere model, as shown by the light curves. It is important to see that both theoretically and experimentally, $\tau_{\Gamma}$ levels off in the limit of $x \rightarrow \infty$ in contrast to the earlier theoretical ${ }^{79}$ and experimental ${ }^{80,81}$ results. Further, it is interesting to see that the dynamic chain stiffness $\left(\tau_{\Gamma} / \tau_{\Gamma}^{0}\right)_{\infty}$ is larger for the chain with larger static chain stiffness $\lambda^{-1}$ (see Table I); there is strong correlation between the two measures.

\section{Dynamic Structure Factor}

According to the HW theory, ${ }^{82}$ the first cumulant $\Omega(k)$ of the dynamic structure factor is given by a sum of two terms,

$$
\eta_{0} \Omega(k) / k_{\mathrm{B}} T k^{3}=[\rho+F(\bar{k})] / 6 \pi \bar{k}
$$

where $\rho$ is the factor defined in eq 8 and $\bar{k}$ is the reduced magnitude of the scattering vector defined by $\bar{k}=$ $\left\langle S^{2}\right\rangle^{1 / 2} k$. The function $F(\bar{k})$ represents the contribution from the internal modes. Thus the so-called "universal" plot of $\eta_{0} \Omega(k) / k_{\mathrm{B}} T k^{3}$ against $\bar{k}$ depends on the kind of polymer, and therefore is not universal.

Figure 18 shows plots of $\eta_{0} \Omega(k) / k_{\mathrm{B}} T k^{3}$ against $\bar{k}$ for a-PS (with $M=4.1 \times 10^{4}-4.4 \times 10^{7}$ ) in cyclohexane at $34.5^{\circ} \mathrm{C}(\Theta)^{83}$ and at $35.0^{\circ} \mathrm{C},{ }^{84}$ a-PMMA (with 


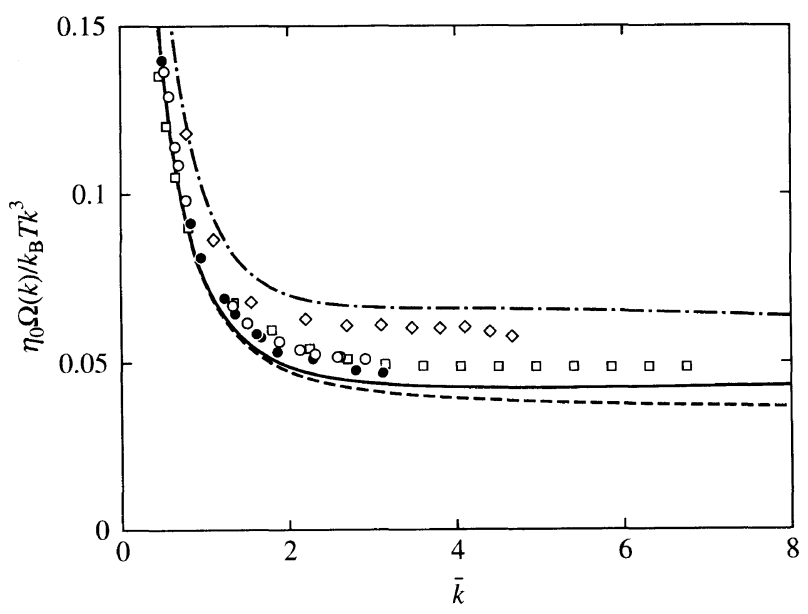

Figure 18. Plots of $\eta_{0} \Omega(k) / k_{\mathrm{B}} T k^{3}$ against $\bar{k}$ for a-PS in cyclohexane at $34.5^{\circ} \mathrm{C}(\Theta)(O)^{83}$ and at $35.0^{\circ} \mathrm{C}(\square),{ }^{84}$ a-PMMA in acetonitrile at $44.0^{\circ} \mathrm{C}(\Theta)(\Theta),{ }^{83}$ and DNA with $L=2.24 \mu \mathrm{m}$ in $0.15 \mathrm{moll}^{-1} \mathrm{NaCl}$ at $25.0^{\circ} \mathrm{C}(\diamond) .^{85}$ The solid, dashed, and dot-dashed curves represent the respective HW or KP theoretical values.

$M=4.86 \times 10^{6}$ and $1.31 \times 10^{7}$ ) in acetonitrile at $44.0^{\circ} \mathrm{C}$ $(\Theta),{ }^{83}$ and DNA with $L=2.24 \mu \mathrm{m}$ in $0.15 \mathrm{moll}^{-1} \mathrm{NaCl}$ at $25.0^{\circ} \mathrm{C}{ }^{85}$ The solid, dashed, and dot-dashed curves represent the respective $\mathrm{HW}$ or KP theoretical values, where the experimental values of $\rho$ have been used in the theoretical calculation. There is rather good agreement between theory and experiment. The complete agreement at small $\bar{k}$ is due to the use of the experimental values of $\rho$, and the underestimate of the theoretical $\Omega(k)$ for the flexible polymers may probably arise from the preaveraging of the Oseen tensor. However, it is important to see that the split of the plots occur at larger $\bar{k}$ (in the plateau region) for the flexible polymers. The indication is that the effects of chain stiffness and local chain conformation (difference in polymer species) on the internal motions are still appreciable even for such large $M$ where the global behavior may be completely described by the Gaussian chain.

\section{CONCLUDING REMARKS}

We have given a brief description of the HW chain and its applications to dilute polymer solutions following most of the chapters of HWCPS. ${ }^{1}$ These developments provide a new framework of polymer solution science, which takes the place of the Flory-Kirkwood framework. It would be possible in the near future to extend the new framework to semidilute solutions.

The final remark is concerned with some applications of the type- 1 generalized KP chain to circular DNA, in particular to the statistical and transport behavior of its topoisomers. Specifically, we can evaluate the ringclosure probabilities, distributions of the linking number and the writhe, translational friction and sedimentation coefficients, and so forth. For the details, the reader is referred to Chapter 7 of HWCPS. ${ }^{1}$

Acknowledgment. The author would like to thank Prof. A. Abe, Editor-in-Chief, for inviting this review article.

\section{REFERENCES AND NOTES}

1. H. Yamakawa, "Helical Wormlike Chains in Polymer Solutions," Springer, Berlin, 1997. Hereafter, this book is referred to as HWCPS.

2. H. Yamakawa, "Modern Theory of Polymer Solutions," Harper \& Row, New York, N.Y., 1971.

3. H. Yamakawa, Ann. Rev. Phys. Chem., 35, 23 (1984).

4. H. Yamakawa, in "Molecular Conformation and Dynamics of Macromolecules in Condensed Systems," M. Nagasawa, Ed., Elsevier, Amsterdam, 1988, p 21.

5. H. Yamakawa, in "Frontiers of Macromolecular Science," $T$. Saegusa, T. Higashimura, and A. Abe, Ed., Blackwell, Oxford, 1989, p 271.

6. H. Yamakawa, Polym. Prepr., Jpn., 36, 1 (1987); 43, 49 (1994).

7. H. Yamakawa, Kasen Koenshu, 51, 9 (1994).

8. P. J. Flory, "Principles of Polymer Chemistry," Cornell Univ. Press, Ithaca, N.Y., 1953.

9. P. J. Flory, "Statistical Mechanics of Chain Molecules," Interscience, New York, N.Y., 1969.

10. H. Yamakawa and M. Fujii, J. Chem. Phys., 64, 5222 (1976).

11. O. Kratky and G. Porod, Rec. Trav. Chim., 68, 1106 (1949).

12. J. G. Kirkwood and J. Riseman, J. Chem. Phys., 16, 565 (1948).

13. J. G. Kirkwood, Rec. Trav. Chim., 68, 649 (1949); J. Polym. Sci., 12, 1 (1954).

14. H. Yamakawa and T. Yoshizaki, J. Chem. Phys., 75, 1016(1981).

15. T. Konishi, T. Yoshizaki, J. Shimada, and H. Yamakawa, Macromolecules, 22, 1921 (1989); and succeeding papers.

16. The term "polymer solution science" is an English translation of the Japanese term "Kobunshi Yoekigaku" due to Yamakawa. This Japanese term was first, although not officially, used by $\mathbf{M}$. Kurata in the mid-1960s. Many of Japanese polymer scientists use the term "Kobunshi Yoekiron" instead of "Kobunshi Yoekigaku."

17. T. Konishi, T. Yoshizaki, T. Saito, Y. Einaga, and H. Yamakawa, Macromolecules, 23, 290 (1990).

18. T. Konishi, T. Yoshizaki, and H. Yamakawa, Macromolecules, 24, 5614 (1991).

19. F. Abe, Y. Einaga, T. Yoshizaki, and H. Yamakawa, Macromolecules, 26, 1884 (1993).

20. Y. Tamai, T. Konishi, Y. Einaga, M. Fujii, and H. Yamakawa, Macromolecules, 23, 4067 (1990).

21. F. Abe, K. Horita, Y. Einaga, and H. Yamakawa, Macromolecules, 27, 725 (1994).

22. M. Kamijo, N. Sawatari, T. Konishi, T. Yoshizaki, and H Yamakawa, Macromolecules, 27, 5697 (1994).

23. M. Kamijo, F. Abe, Y. Einaga, and H.Yamakawa, Macromolecules, 28, 1095 (1995).

24. M. R. Ambler, D. McIntyre, and L. J. Fetters, Macromolecules, 11, 300 (1978).

25. Y. Kashiwagi, T. Norisuye, and H. Fujita, Macromolecules, 14, 1220 (1981).

26. M. Yamada, M. Osa, T. Yoshizaki, and H. Yamakawa, Macromolecules, 30, 7166 (1997).

27. J. E. Godfrey and H. Eisenberg, Biophys. Chem., 5, 301 (1976).

28. H. Koyama, T. Yoshizaki, Y. Einaga, H. Hayashi, and $\mathrm{H}$ Yamakawa, Macromolecules, 24, 932 (1991).

29. T. Yoshizaki, H. Hayashi, and H. Yamakawa, Macromolecules, 26, 4037 (1993).

30. K. Horita, T. Yoshizaki, H. Hayashi, and H. Yamakawa, Macromolecules, 27, 6492 (1994).

31. T. Yoshizaki, H. Hayashi, and H. Yamakawa, Macromolecules, 27, 4259 (1994).

32. R. G. Kirste and W. Wunderlich, Makromol. Chem., 73, 240 (1964).

33. W. Wunderlich and R. G. Kirste, Ber. Bunsen-Ges. Phys. Chem., 68, 646 (1964)

34. D. Y. Yoon and P. J. Flory, Macromolecules, 9, 299 (1976).

35. K. Nagasaka, T. Yoshizaki, J. Shimada, and H. Yamakawa, Macromolecules, 24, 924 (1991)

36. Y. Takaeda, T. Yoshizaki, and H. Yamakawa, Macromolecules, 26, 3742 (1993)

37. Y. Takaeda, T. Yoshizaki, and H. Yamakawa, Macromolecules, 28, 4167 (1995).

38. H. Ando, T. Yoshizaki, A. Aoki, and H. Yamakawa, Macro- 
molecules, 30, 6199 (1997).

39. T. Yamada, T. Yoshizaki, and H. Yamakawa, Macromolecules, 25, 1487 (1992).

40. H. Yamakawa and T. Yoshizaki, Macromolecules, 15, 1444 (1982).

41. Y. Einaga, H. Koyama, T. Konishi, and H. Yamakawa, Macromolecules, 22, 3419 (1989).

42. F. Abe, Y. Einaga, and H. Yamakawa, Macromolecules, 26, 1891 (1993)

43. Y. Fujii, Y. Tamai, T. Konishi, and H. Yamakawa, Macromolecules, 24, 1608 (1991).

44. F. Abe, Y. Einaga, and H. Yamakawa, Macromolecules, 24, 4423 (1991).

45. T. Yamada, H. Koyama, T. Yoshizaki, Y. Einaga, and $\mathrm{H}$. Yamakawa, Macromolecules, 26, 2566 (1993).

46. H. Murakami, T. Norisuye, and H. Fujita, Macromolecules, 13, 345 (1980).

47. T. Yanaki, T. Norisuye, and H. Fujita, Macromolecules, 13, 1462 (1980).

48. T. Yamada, T. Yoshizaki, and H. Yamakawa, Macromolecules, 25, 377 (1992)

49. K. Dehara, T. Yoshizaki, and H. Yamakawa, Macromolecules, 26, 5137 (1993)

50. T. Arai, N. Sawatari, T. Yoshizaki, Y. Einaga, and H. Yamakawa, Macromolecules, 29, 2309 (1996).

51. B. H. Zimm, J. Chem. Phys., 24, 269 (1956)

52. H. Yamakawa and T. Yoshizaki, J. Chem. Phys., 91, 7900 (1989).

53. H. Yamakawa and W. H. Stockmayer, J. Chem. Phys., 57, 2843 (1972).

54. H. Yamakawa and J. Shimada, J. Chem. Phys., 83, 2607 (1985)

55. J. Shimada and H. Yamakawa, J. Chem. Phys., 85, 591 (1986).

56. C. Domb and A. J. Barrett, Polymer, 17, 179 (1976).

57. K. Horita, F. Abe, Y. Einaga, and H. Yamakawa, Macromolecules, 26, 5067 (1993).

58. K. Horita, N. Sawatari, T. Yoshizaki, Y. Einaga, and H. Yamakawa, Macromolecules, 28, 4455 (1995).

59. A. J. Barrett, Macromolecules, 17, 1561 (1984); 17, 1566 (1984).

60. H. Yamakawa and T. Yoshizaki, Macromolecules, 28, 3604 (1995).

61. T. Yoshizaki and H. Yamakawa, J. Chem. Phys., 105, 5618 (1996).

62. H. Yamakawa, Macromolecules, 25, 1912 (1992)
63. Y. Einaga, F. Abe, and H. Yamakawa, Macromolecules, 26, 6243 (1993).

64. F. Abe, Y. Einaga, and H. Yamakawa, Macromolecules, 27, 3262 (1994).

65. Y. Miyaki, Ph. D. Thesis, Osaka Univ., Osaka, Japan, 1981.

66. H. Yamakawa, F. Abe, and Y. Einaga, Macromolecules, 26, 1898 (1993).

67. H. Yamakawa, Macromolecules, 26, 5061 (1993).

68. H. Yamakawa, F. Abe, and Y. Einaga, Macromolecules, 27, 5704 (1994).

69. F. Abe, Y. Einaga, and H. Yamakawa, Macromolecules, 28, 694 (1995).

70. M. Yamada, T. Yoshizaki, and H. Yamakawa, Macromolecules, 31, 7728 (1998).

71. T. Norisuye, Y. Nakamura, and K. Akasaka, Macromolecules, 26, 3791 (1993).

72. T. Norisuye and Y. Nakamura, Macromolecules, 27, 2054 (1994)

73. H. Yamakawa, F. Abe, and Y. Einaga, Macromolecules, 27, 3272 (1994).

74. T. Yoshizaki and H. Yamakawa, J. Chem. Phys., 104, 1120 (1996).

75. M. Fixman and J. Kovac, J. Chem. Phys., 61, 4939 (1974).

76. Y. Takaeda, T. Yoshizaki, and H. Yamakawa, Macromolecules, 27, 4248 (1994)

77. Y. Takaeda, T. Yoshizaki, and H. Yamakawa, Macromolecules, 28, 682 (1995).

78. Y. Naito, N. Sawatari, Y. Takaeda, T. Yoshizaki, and H. Yamakawa, Macromolecules, 30, 2751 (1997).

79. K. Ono and K. Okano, Japan J. Appl. Phys., 9, 1356 (1970).

80. D. R. Bauer, J. I. Brauman, and R. Pecora, Macromolecules, 8, 443 (1975).

81. F. Strehle, Th. Dorfmüller, and D. Samios, Macromolecules, 25, 3569 (1992)

82. T. Yoshizaki, M. Osa, and H. Yamakawa, J. Chem. Phys., 106, 2828 (1997).

83. N. Sawatari, T. Yoshizaki, and H. Yamakawa, Macromolecules, 31, 4218 (1998).

84. C. C. Han and A. Z. Akcasu, Macromolecules, 14, 1080 (1981).

85. K. Soda and A. Wada, Biophys. Chem., 20, 185 (1984). 